\title{
Bevacizumab treatment for symptomatic spinal ependymomas in neurofibromatosis type 2
}

\section{Citation}

Farschtschi, S., V. L. Merker, D. Wolf, M. Schuhmann, J. Blakeley, S. R. Plotkin, C. Hagel, and V. F. Mautner. 2015. "Bevacizumab Treatment for Symptomatic Spinal Ependymomas in Neurofibromatosis Type 2." Acta Neurologica Scandinavica 133 (6) (September 15): 475-480. doi:10.1111/ane.12490.

\section{Published Version}

doi:10.1111/ane.12490

\section{Permanent link}

http://nrs.harvard.edu/urn-3:HUL.InstRepos:33788489

\section{Terms of Use}

This article was downloaded from Harvard University's DASH repository, and is made available under the terms and conditions applicable to Open Access Policy Articles, as set forth at http:// nrs.harvard.edu/urn-3:HUL.InstRepos:dash.current.terms-of-use\#OAP

\section{Share Your Story}

The Harvard community has made this article openly available.

Please share how this access benefits you. Submit a story.

\section{Accessibility}




\section{Bevacizumab Treatment for Symptomatic Spinal Ependymomas in Neurofibromatosis}

\section{Type 2}

S. Farschtschi ${ }^{1}$ MD, V.L. Merker ${ }^{2}$, D. Wolf ${ }^{3}$ MD, M. Schuhmann ${ }^{4}$ MD, ${ }^{\dagger J}$. Blakeley ${ }^{3}$ MD, ${ }^{\ddagger}$ S.R. Plotkin $^{2} \mathrm{MD}$ PhD, ${ }^{\ddagger} \mathrm{C}$. Hagel $^{5} \mathrm{MD},{ }^{\ddagger}$ V.F. Mautner ${ }^{1} \mathrm{MD}$

These four authors contributed equally to the publication

Affiliations:

1. University Medical Center Hamburg-Eppendorf, Department of Neurology, Martinistr. 52, 20246 Hamburg, Germany

2. Massachusetts General Hospital, Pappas Center for Neuro Oncology. 55 Fruit Street, Boston, MA 02114, USA

3. The Johns Hopkins Hospital, Department of Neurology, 1550 Orleans Street, Baltimore, MD 21231, USA

4. University Medical Center Tübingen, Department of Neurosurgery, Hoppe-Seyler-Str. 3, 72076 Tübingen, Germany

5. University Medical Center Hamburg-Eppendorf, Institute of Neuropathology, Martinistr. 52, 20246 Hamburg, Germany

Corresponding Author:

Said Farschtschi

Martinistr. 52

20246 Hamburg

Germany

Phone: $+4940-7410-55629$

Fax: $+4940-7410-55966$

E-mail: s.farschtschi@uke.de

Word Count: 1490

Keywords: Neurofibromatosis, ependymoma, bevacizumab, NF2, VEGF

Short Title: Bevacizumab for Symptomatic Spinal Ependymomas in NF2 


\begin{abstract}
Background: Neurofibromatosis type 2 (NF2) is a tumor suppressor syndrome associated with vestibular schwannomas, meningiomas, and spinal ependymomas. There have been anecdotal reports of radiographic response of spinal ependymomas in NF2 patients being treated for progressive vestibular schwannomas with bevacizumab, a monoclonal antibody against vascular endothelial growth factor (VEGF).

Aims: To review the clinical effects of bevacizumab treatment for symptomatic, NF2-associated ependymomas

Methods: We conducted a retrospective review of all patients with NF2 treated with bevacizumab for symptomatic ependymoma at three NF2 specialty centers. Tumor size was evaluated by linear measurements; radiographic response was defined as $>20 \%$ reduction in tumor size. We also performed immunohistochemical evaluation of NF2-associated symptomatic ependymomas from 5 patients, including 2 from this clinical series.

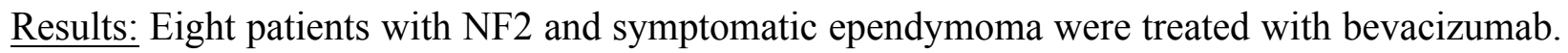
All patients had subjective clinical improvement with bevacizumab, although only $5 / 8$ patients evaluated had radiographic response. All tumors expressed VEGF-R2. 4/5 evaluated ependymomas expressed VEGF-R1; one without VEGF-R1 expression was from a patient who showed clinical but not radiographic response.

Conclusions: Treatment using bevacizumab improved symptoms related to NF2-associated ependymomas, often without concurrent radiographic response. This treatment effect may be related to VEGF-R1 expression in NF2-associated ependymoma.
\end{abstract}




\section{Introduction}

Neurofibromatosis type 2 (NF2) is a neurogenetic tumor predisposition disorder with an incidence of $\sim 1: 27,000$. Hallmark lesions of NF2 include bilateral vestibular schwannomas, nonvestibular schwannomas and meningiomas. In addition, an estimated 33\% of patients with NF2 have spinal ependymomas (4). Histopathology of these lesions includes tanycytic and myxopapillary (WHO grade 1) and classic (WHO grade 2) ependymoma; signs of anaplasia are uncommon (4;5). In contrast to their sporadic counterparts, ependymomas in NF2 appear almost exclusively in the spinal cord and are characterized by multi-segment tumor formation with large cystic compartments.

In most NF2 patients, spinal ependymomas are asymptomatic and therapy is not required; however, in about one-fourth of patients with ependymomas, tumors can cause significant neurological symptoms. Currently, the most common treatment for NF2-related symptomatic ependymomas is surgical resection. However, surgery has a high risk of spinal cord injury and perioperative complications, especially in patients exhibiting severe neurological symptoms such as paraparesis or sensory loss prior to surgery (4). For this reason, chemotherapeutic options for treatment are highly desirable.

Recent investigations have revealed potentially promising effects of bevacizumab on NF2-related vestibular schwannomas (9). Bevacizumab is a recombinant humanized monoclonal antibody against vascular endothelial growth factor (VEGF-A). Neutralization of VEGF may lead to inhibition of tumor growth, decreases in interstitial fluid pressure, and increased tumor oxygenation.

The effect of bevacizumab on ependymomas has not been established. In a small case series, $87.5 \%$ of adults with sporadic, recurrent ependymoma achieved either a partial response 
or stable disease after treatment with bevacizumab (alone or in combination with cytotoxic chemotherapy) (11). However, in sporadic recurrent ependymoma in children, bevacizumab and irinotecan failed to show any sustained response (12). A report on the effect of bevacizumab in NF2 patients being treated for progressive vestibular schwannoma suggested an effect on spinal ependymomas (13), but to date, no studies have systematically addressed the response of symptomatic spinal ependymomas to bevacizumab in NF2. Here we report the clinical effect of bevacizumab on ependymomas in 8 patients with NF2, and VEGF receptor expression in 5 resected, NF2-related ependymomas.

\section{Methods}

All patients were diagnosed with NF2 by experienced clinicians based on consensus criteria. Patients received bevacizumab as treatment for symptomatic ependymoma after consulting neurosurgeons deemed them poor candidates for surgery or they refused surgical treatment. Using MRIs of the head and spine performed with intravenous gadolinium contrast, we measured ependymoma size (in $\mathrm{mm}$ ) using T2-weighted images with radiographic response was defined as $\geq 20 \%$ reduction in linear measures. Volumetric assessment of ependymomas was not feasible due to the inhomogeneous appearance and cystic tumor formation. We measured vestibular schwannoma volume (in $\mathrm{cm}^{3}$ ) using $\mathrm{T} 1$ post-contrast images, with radiographic response defined as $\geq 20 \%$ reduction in volume. Clinical and radiological follow up were performed routinely every 6 to 12 months.

Immunohistochemistry: Three NF2-associated ependymomas from a case series previously reported by Hagel et al. (5) were available for immunohistochemical evaluation of VEGF-R1 and 
VEGF-R2. In addition, pre-treatment surgical specimens from patients 3 and 5 of this case series were also analyzed for VEGF-R1 and R2. Immunohistochemical studies used four micrometer thick sections. Tissue sections were heat pre-treated followed by incubation with VEGFR-1 or VEGFR-2 antibodies (Santa Cruz sc-316, 1:100; Santa Cruz sc-504, 1:50) in an automated stainer (Ventana Medical Systems, Tucson, AZ, USA) according to standard protocols. Bound antibodies were detected by the peroxidase method using diaminobenzidine as chromogen (760500, Ultraview DAB, Ventana). All antibody reactions were validated prior to the study using isotype negative-controls and glioblastoma samples harboring pathological endothelial proliferations as positive controls (data not shown).

\section{Results}

3.1 Patient characteristics: 8 patients ( 7 male, 1 female) from three institutions were treated with bevacizumab for symptomatic spinal ependymomas (Table 1). Median age at time of treatment was 22 years (range 15-34 years). Symptoms prompting treatment included dysphagia, ataxic gait, spastic paraparesis and intractable pain. Two patients underwent surgery prior to bevacizumab treatment (patient 3 and 5), with pathological confirmation of the diagnosis of ependymoma; for all other patients, diagnosis of ependymoma was made based on characteristic radiographic appearance. Seven patients had radiographically documented ependymoma growth prior to treatment.

3.2 Bevacizumab treatment: The initial dose of bevacizumab was either 5 or $7.5 \mathrm{mg}$ per kg every 2 weeks. In some patients, the dose was decreased during the course of treatment to minimize long term toxicity; four patients received $3.75 \mathrm{mg} / \mathrm{kg}$ every two weeks and four 
patients received $7.5-10 \mathrm{mg} / \mathrm{kg}$ every three weeks. The median duration of treatment was 13.5 months (range 6-58 months).

3.3 Clinical and radiographic response: All eight patients had subjective improvement in neurologic function as rated both by the patient and the care team. Improved neurological symptoms included dysphagia (\#1-2), sensory deficits (\#3-8), weakness (\#3-6), bladder and bowel dysfunction (\#3), pain (\#3, 6-8), and ataxia (\#4-6).

Half of patients experienced radiographic response of ependymoma, seen within 3 to 6 months after treatment initiation. In all four patients with a radiographic response (\#4-7), the cystic compartments of the ependymoma demonstrated the greatest decrease in size. Importantly, there were no instances of pseudoresponse - the resolution of contrast enhancement - noted in any patients. (14) There was no obvious relationship between the degree of tumor shrinkage and perceived clinical benefit. For example, patient 3, who showed no significant radiographic change in tumor size, demonstrated improvement of ataxia, paraparesis and urinary incontinence such that he regained bladder control and ambulation.

3.4 Treatment breaks: In $5 / 8$ patients, treatment was held for $\geq 6$ months. In three patients (\#1,4,\& 7), symptomatic improvements noted during treatment were lost during treatment hold, but returned within a few months when drug was re-started. Radiographic progression was noted in patient 4 during treatment hold (Fig. 1), but was followed by a reduction of tumor size to the baseline volume after restarting bevacizumab. After stopping treatment, patient 2 and 8 presented with radiological relapse and increasing back pain.

3.5 Toxicity: Toxicity related to bevacizumab treatment was mild. One patient had a single episode of abdominal pain with hematochezia for which bevacizumab was held for 2 
months. Symptoms improved, work-up was negative, and he was able to resume drug without complication. There were no grade 3 or 4 adverse events related to bevacizumab.

3.6 Immunohistochemistry: Upon immunohistochemical investigation, all tumors investigated showed expression of VEGF-R2 in all parts of the tumor (Fig. 2). 4/5 tumors showed expression of VEGFR-1 in tumor cells, and in 2/5 cases, the receptor was detected in endothelial cells. Staining ranged from scattered single cells over patchy expression to strong labeling of large tumor areas (Fig. 3). In one case, (patient 3) the staining was negative. This patient experienced clinical improvement but no radiographic response.

\section{Discussion}

This is the first case series to investigate the effect of bevacizumab on symptomatic spinal ependymomas in patients with NF2. All eight patients showed clinical improvement, despite significant neurological impairments at baseline. Continued treatment with bevacizumab was necessary to maintain neurological improvement. In our experience, clinical and radiographic improvement occurred within 3 to 6 months of treatment and clinical relapse occurred about 6 months after treatment discontinuation. Interestingly, some individuals had discordant radiographic changes in vestibular schwannomas and ependymoma, pointing to the heterogeinity of NF2-related tumors.

Shrinkage of tumors was primarily noted in the cystic portions of ependymomas, suggesting that the primary effect of bevacizumab is on vascular permeability rather than on tumor cells. The clinical improvement after treatment onset may be explained by an anti-edema effect of bevacizumab. In previous studies on vestibular schwannoma treated with bevacizumab, 
there was a correlation between tumor shrinkage and change in the apparent diffusion coefficient value, a radiological marker for edema (8).

We found a slight endothelial expression and strong tumor tissue expression of VEGFR-1 in 4/5 NF2-associated spinal ependymomas. Our findings agree with that of Chan et al. (15), who reported that $5 / 6$ sporadic ependymomas investigated by in-situ-hybridization for VEGFR-1 and VEGFR-2 expression had slight to strong expression of mRNA of both receptors.

From our case series, patient 3 showed no significant expression of VEGF-R1 prior to treatment, which could explain the lack of radiographic response in this patient. However, this patient still displayed significant clinical improvement despite the lack of tumor shrinkage. For this reason, we believe that clinical improvement, rather than radiographic response, should be used to assess the benefit of bevacizumab for patients with NF2.

Because of the small sample size, our data cannot be generalized to all NF2 patients with symptomatic spinal ependymomas. Ependymomas were evaluated by linear measures and not by volumetric measurement, so small changes in tumor size might not have been detected. The patients presented in this case series were selected for bevacizumab treatment due to severe symptoms associated with their spinal ependymomas; however, many NF2 patients with ependymomas have few or no clinical impairments, making drug treatment unnecessary.

Taken together, these findings suggest bevacizumab improves clinical symptoms related to NF2-related spinal ependymoma in selected patients, with a less robust effect on tumor size. Further investigation is warranted to study the mechanisms and potential benefit of bevacizumab as a novel option for treatment of symptomatic ependymomas in NF2 patients.

Conflicts of interest: The authors declare they have no conflicts of interest. 
Sources of funding: No funding

Acknowledgment: None

Declaration: This study was conducted in full accordance with the declaration of Helsinki. 


\section{Reference List}

(1) Evans DG, Moran A, King A, Saeed S, Gurusinghe N, Ramsden R. Incidence of vestibular schwannoma and neurofibromatosis 2 in the North West of England over a 10year period: higher incidence than previously thought. Otol Neurotol 2005 Jan;26(1):937.

(2) Mautner VF, Lindenau M, Baser ME, Hazim W, Tatagiba M, Haase W, et al. The neuroimaging and clinical spectrum of neurofibromatosis 2. Neurosurgery 1996 May;38(5):880-5.

(3) Mautner VF, Tatagiba M, Lindenau M, Funsterer C, Pulst SM, Baser ME, et al. Spinal tumors in patients with neurofibromatosis type 2: MR imaging study of frequency, multiplicity, and variety. AJR Am J Roentgenol 1995 Oct;165(4):951-5.

(4) Plotkin SR, O'Donnell CC, Curry WT, Bove CM, MacCollin M, Nunes FP. Spinal ependymomas in neurofibromatosis Type 2: a retrospective analysis of 55 patients. $\mathrm{J}$ Neurosurg Spine 2011 Apr;14(4):543-7.

(5) Hagel C, Stemmer-Rachamimov AO, Bornemann A, Schuhmann M, Nagel C, Huson S, et al. Clinical presentation, immunohistochemistry and electron microscopy indicate neurofibromatosis type 2-associated gliomas to be spinal ependymomas. Neuropathology 2012 Dec;32(6):611-6.

(6) Lee SH, Chung CK, Kim CH, Yoon SH, Hyun SJ, Kim KJ, et al. Long-term outcomes of surgical resection with or without adjuvant radiation therapy for treatment of spinal ependymoma: a retrospective multicenter study by the Korea Spinal Oncology Research Group. Neuro Oncol 2013 Jul;15(7):921-9.

(7) Epstein FJ, Farmer JP, Freed D. Adult intramedullary spinal cord ependymomas: the result of surgery in 38 patients. J Neurosurg 1993 Aug;79(2):204-9.

(8) Plotkin SR, Stemmer-Rachamimov AO, Barker FG, Halpin C, Padera TP, Tyrrell A, et al. Hearing improvement after bevacizumab in patients with neurofibromatosis type $2 . \mathrm{N}$ Engl J Med 2009 Jul 23;361(4):358-67.

(9) Plotkin SR, Merker VL, Halpin C, Jennings D, McKenna MJ, Harris GJ, et al. Bevacizumab for progressive vestibular schwannoma in neurofibromatosis type 2: a retrospective review of 31 patients. Otol Neurotol 2012 Aug;33(6):1046-52.

(10) Jain RK. Antiangiogenic therapy for cancer: current and emerging concepts. Oncology (Williston Park) 2005 Apr;19(4 Suppl 3):7-16.

(11) Green RM, Cloughesy TF, Stupp R, DeAngelis LM, Woyshner EA, Ney DE, et al. Bevacizumab for recurrent ependymoma. Neurology 2009 Nov 17;73(20):1677-80. 
(12) Gururangan S, Fangusaro J, Young PT, Onar-Thomas A, Gilbertson RJ, Vajapeyam S, et al. Lack of efficacy of bevacizumab + irinotecan in cases of pediatric recurrent ependymoma--a Pediatric Brain Tumor Consortium study. Neuro Oncol 2012 Nov;14(11):1404-12.

(13) Mautner VF, Nguyen R, Kutta H, Fuensterer C, Bokemeyer C, Hagel C, et al. Bevacizumab induces regression of vestibular schwannomas in patients with neurofibromatosis type 2. Neuro Oncol 2010 Jan;12(1):14-8.

(14) van den Bent MJ, Wefel JS, Schiff D, Taphoorn MJ, Jaeckle K, Junck L, et al. Response assessment in neuro-oncology (a report of the RANO group): assessment of outcome in trials of diffuse low-grade gliomas. Lancet Oncol 2011 Jun;12(6):583-93.

(15) Chan AS, Leung SY, Wong MP, Yuen ST, Cheung N, Fan YW, et al. Expression of vascular endothelial growth factor and its receptors in the anaplastic progression of astrocytoma, oligodendroglioma, and ependymoma. Am J Surg Pathol 1998

Jul;22(7):816-26. 
Table 1. Patient characteristics

\begin{tabular}{|l|l|l|l|l|l|l|l|l|l|l|l|}
\hline & & & & & & & & & \\
\end{tabular}

m: male, f: female, q3: every three weeks, q2: every two weeks 
${ }^{1}$ In addition to symptomatic ependymoma, patient also had progressive vestibular schwannoma.

${ }^{2}$ Imunnohistochemical staining of pre-treatment surgical specimen revealed the ependymoma was negative for VEGFR-1 and positive for VERGFR-2.

${ }^{3}$ Imunnohistochemical staining of pre-treatment surgical specimen revealed the ependymoma was positive for both VEGFR-1 and VEGFR-2. 
Fig. 1: Spinal ependymoma (SE) in the cervical cord with a large cystic compartment (cy) of patient 4, shown with T2-weighted sagittal MRI (Siemens Avanto 1.5T).

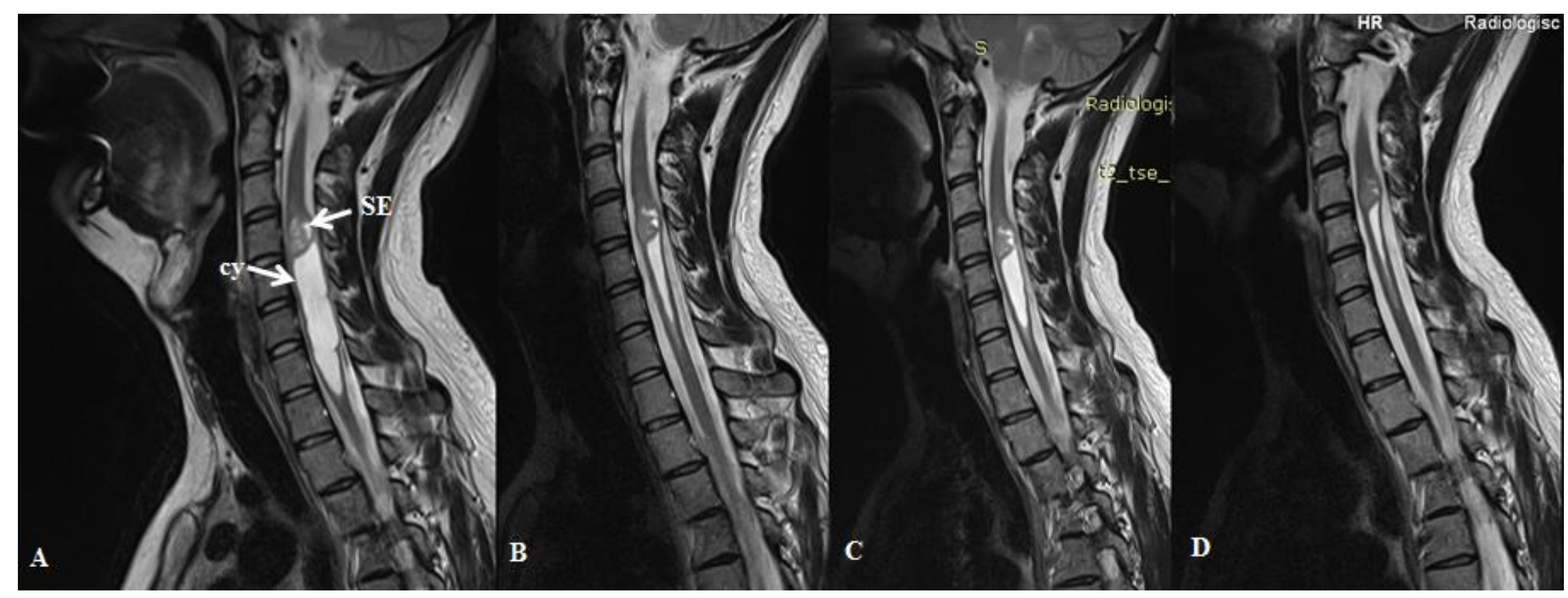

A) Before treatment with bevacizumab. B) After 6 months of treatment. C) 6 month after discontinuation of treatment. D) After retreatment. 
Fig. 2: Immunohistochemical demonstration of VEGFR2 in spinal ependymoma of NF2 patients.

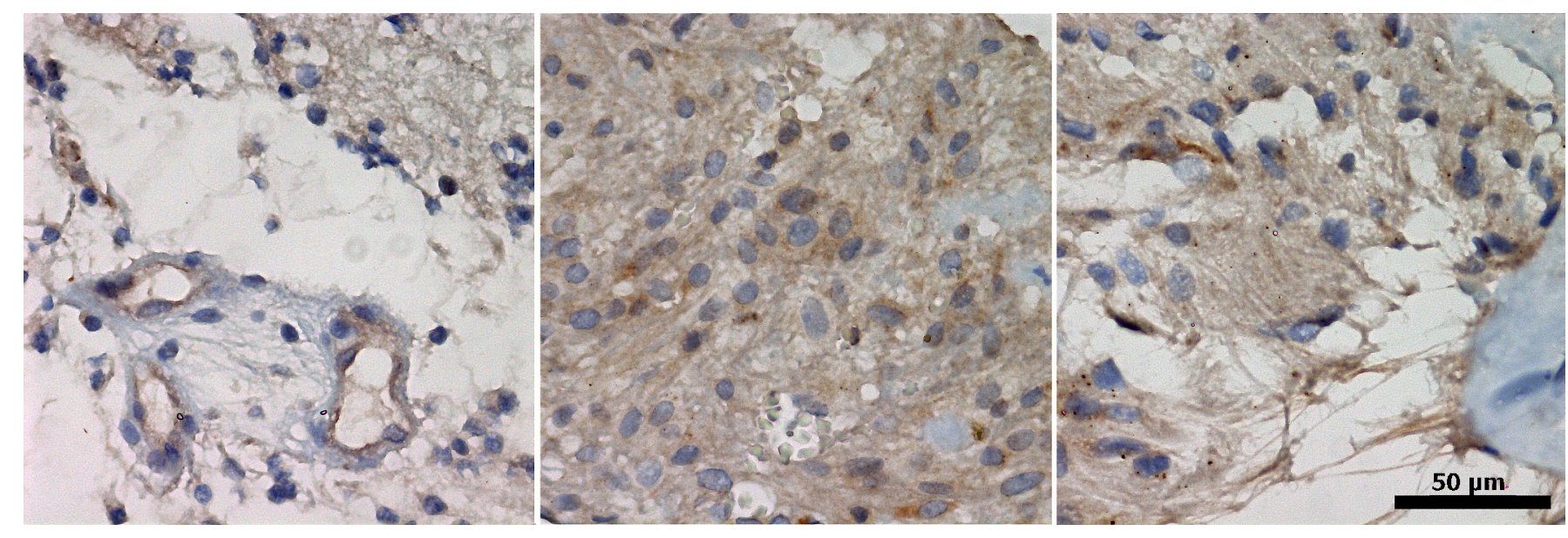

Varying expression of VEGF-R2 in three samples of NF2-associated ependymomas, both in the vasculature and the tumor tissue. 
Fig. 3: Immunohistochemical demonstration of VEGFR-1 expression in spinal ependymoma of NF2 patients.

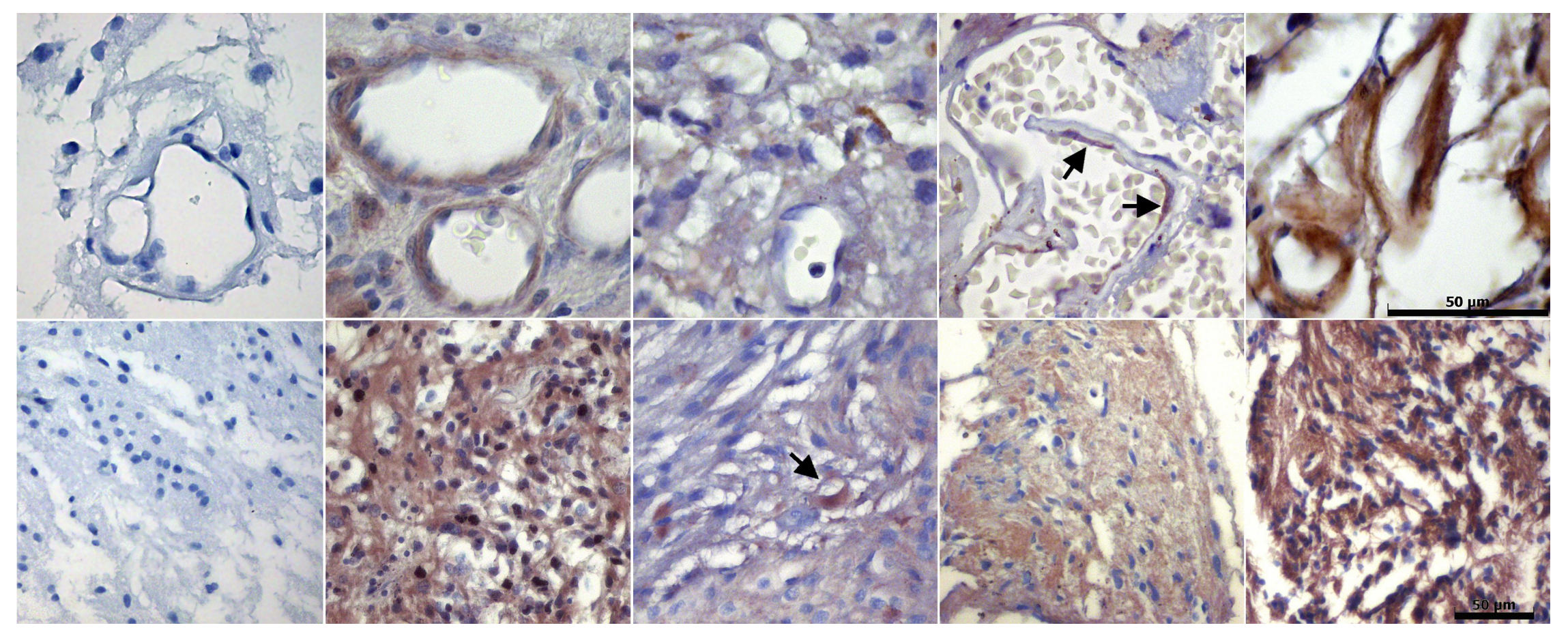

From left to right, tumor of patient 3, tumor of patient 5, three additional NF2-associated ependymomas from another case series;

upper row, expression of VEGF-receptor in tumor endothelia; lower row, expression of VEGF-receptor in the tumor tissue; arrows indicate scattered positive cells. 